\title{
EchoGéo
}

25 | 2013

Moyen-Orient : espaces et passeurs du changement

\section{Le Moyen-Orient sans orientalisme}

Jean-Louis Chaléard

\section{(2) OpenEdition}

Journals

Édition électronique

URL : https://journals.openedition.org/echogeo/13578

DOI : 10.4000/echogeo.13578

ISSN : 1963-1197

\section{Éditeur}

Pôle de recherche pour l'organisation et la diffusion de l'information géographique (CNRS UMR 8586)

Référence électronique

Jean-Louis Chaléard, «Le Moyen-Orient sans orientalisme », EchoGéo [En ligne], 25 | 2013, mis en ligne le 10 octobre 2013, consulté le 11 août 2021. URL : http://journals.openedition.org/echogeo/13578 ; DOI : https://doi.org/10.4000/echogeo.13578

Ce document a été généré automatiquement le 11 août 2021.

EchoGéo est mis à disposition selon les termes de la licence Creative Commons Attribution - Pas d'Utilisation Commerciale - Pas de Modification 4.0 International (CC BY-NC-ND) 


\title{
Le Moyen-Orient sans orientalisme
}

\author{
Jean-Louis Chaléard
}

1 On se souvient de la célèbre phrase du Général De Gaulle : « Vers l'Orient compliqué, je volais avec des idées simples " (Mémoires de guerre). La présente livraison d'Échogéo est largement consacrée au Proche et au Moyen-Orient, puisque toutes les rubriques du numéro sont, plus ou moins, concernées par des questions afférant à cet espace. Des sujets très divers sont abordés, allant de la question (classique) de l'eau à des domaines moins courants en géographie comme ceux touchant à la sexualité, introduisant la complexité des situations territoriales et des rapports sociaux dans ces régions et renvoyant de cet Orient une image qui n'a rien de « simple ».

Doit-on insister sur la singularité d'un espace au demeurant mal défini ? Les notions de «Proche-Orient » et de " Moyen-Orient », comme le souligne Serge Weber, nécessitent une distance critique, tant elles apparaissent comme l'émanation de découpages européocentrés et datés. Les articles abordent des terrains allant de l'Égypte à l'Iran, en passant par le Golfe, les Kurdistan, etc. sans que des bornes précises soient fixées. L'inclusion d'Israël contribue à casser l'image d'Épinal d'un Orient arabe et musulman. Pour autant, des questionnements communs émergent. Ils renvoient souvent à des interrogations qui dépassent la région: ce sont particulièrement celles au cœur des analyses de géographie urbaine, sur les espaces publics notamment. Mais se dessinent aussi des problématiques propres ou des configurations régionales de questionnements généraux sur l'actualité, l'individu, les relations entre pouvoirs et populations urbaines...

3 Le dossier de la rubrique Sur le champ, dirigé par Serge Weber, porte sur «Espaces et passeurs de changement au Moyen-Orient». Les huit articles présentés étudient la question $\mathrm{du}$ "transnational au-delà du nationalisme». Les révolutions récentes, abordées directement ou indirectement dans plusieurs textes (Égypte...), mettent en évidence la circulation des idées transfrontalières, que l'on peut percevoir concrètement à travers divers exemples (ne serait-ce que dans les migrations vers les pays du Golfe). Comme le souligne Serge Weber, les constructions nationales se sont souvent faites à l'encontre des réalités sociales locales multiples et complexes (religieuses, de classes, etc.) : le national, affirmé avec force et/ou par la force vient 
souvent d'idéologies qui dépassent la nation, comme le panarabisme ou la modernisation laïque à l'« occidentale ».

4 Le transnational est analysé de différentes façons. D’abord à travers des thèmes régionaux qui posent des questions centrales pour cet ensemble : l'eau et le pétrole (sujet du premier article), les réalités transfrontalières ou les mobilités (comme les circulations à la frontière entre Kurdes d'Irak et d'Iran). Sont ainsi soulignées la diversité des populations, l'absence d'adéquation entre peuples et frontières d'État, résultat souvent d'un découpage discutable après la première Guerre mondiale, l'inégale distribution des ressources au sujet de laquelle peu de choses sont faites pour y remédier, etc.

5 Un des intérêts du dossier est aussi de décaler le regard vers « le bas » et les « marges », qu'elles soient géographiques ou sociales, ce qui permet une approche approfondie et différente des réalités. Les "marges", ce sont bien sûr les périphéries, les régions frontalières, mais aussi les espaces des minorités sexuelles ou sociales (comme les espaces de l'homosexualité à Beyrouth). Les articles sur les rapports amoureux, la sexualité, sont l'occasion de réfléchir sur les assignations de genre pour les femmes, les contraintes vestimentaires, etc. Au-delà, c'est une géographie du quotidien qui se dessine à mi-chemin entre une analyse des comportements de groupes ou de catégories sociales (les jeunes entre autres) et une réflexion sur les territoires.

6 Ce n'est pas la première fois qu'Échogéo présente des dossiers fondés sur une approche pluridisciplinaire. Dans le présent numéro, les géographes sont certes nombreux, mais on trouve aussi des textes d'anthropologues, de sociologues, etc. Leurs voix se mêlent pour traduire une réalité complexe. Ainsi, l'anthropologie du flirt émirati, à travers l'analyse des stratégies des jeunes amoureux, dessine une géographie de l'espace public et de la ville dans laquelle les shopping malls deviennent des lieux de tolérance pour les jeunes couples.

7 Les autres rubriques du présent numéro complètent et enrichissent le dossier. Dans la rubrique Sur le vif, un article nous mène au cœur de la contestation turque qui a secoué particulièrement Istanbul et fait écho aux évocations des autres révolutions tandis que celui sur le (les ?) Kurdistan renvoie aux problèmes nés des découpages frontaliers, aux marges et au «transnational ». La rubrique Sur l'écrit est particulièrement riche dans cette livraison avec quatre articles ou compte rendus qui abordent des thèmes variés. Thèmes politiques (à travers la présentation de l'Atlas du Proche-orient arabe, ou de l'interview de Kerem Öktem qui décrit la situation turque au Proche-Orient), thèmes politiques et sociaux (à partir de l'analyse du roman Utopia, qui montre les profondes divisions de la société égyptienne) ou plus sociaux (à travers ce qui touche à la sexualité dans le texte sur les revues libanaises) mais qui tous renvoient là-encore aux thèmes du dossier de la rubrique Sur le champ.

Dans l'ensemble, les articles présentés dans cette livraison relèvent d'une approche qui se situe très loin du regard dominateur occidental longtemps porté sur la région et dénoncé par Edward W. Said (dans L'Orientalisme). Ils révèlent un espace multiple, fait de contradictions, à partir de l'analyse des singularités régionales et individuelles. Ils permettent de mieux comprendre certaines questions d'actualité comme d'entrer dans des analyses fines de territoires transnationaux ou intra-urbains.

9 Seul l'article de la rubrique Sur vif consacré à la Chine échappe dans ce numéro au tropisme moyen-oriental. Il s'en échappe à la fois par l'espace en jeu et par sa nature : il s'agit de la conférence faite par Thierry Sanjuan en ouverture du festival de géographie 
de Saint-Dié dont le pays invité était cette année la Chine. Nous avons déjà publié des communications à des ateliers ou colloques lorsque celles-ci nous semblaient dignes d'intérêt. Ce texte nous a paru intéressant parce qu'il fait le point sur une des grandes puissances du monde actuel et permet de réfléchir autant sur la nouvelle place de la Chine dans le concert des nations que sur les processus d'émergence qui touchent un certain nombre de pays qu'on appelait autrefois "tiers monde " et qu'on désigne souvent aujourd'hui sous l'appellation de "Sud». Mais comme pour les articles sur le Moyen-Orient, on pourrait revenir sur la distance qui sépare les analyses actuelles de ce qui fut la tradition de l'École française d'Extrême Orient et que Edward W. Said, au fond, ne distingue pas vraiment de la vision occidentale de l'Orientalisme. Les approches récentes nous donnent ainsi à voir une nouvelle géographie, moins européocentrée et qui répond à l'évolution du monde contemporain lui-même. 